\title{
The Effect of 6-Mercaptopurine on Natural Killer-Cell Activities in Crohn's Disease
}

\author{
M. BROGAN,${ }^{1}$ J. HISERODT, ${ }^{2}$ M. OLIVER, ${ }^{1}$ R. STEVENS,${ }^{1}$ B. KORELITZ, ${ }^{3}$ and S. TARGAN ${ }^{1,4}$
}

Accepted: February 14, 1985

Crohn's disease patients on long-term 6-mercaptopurine therapy (more than 4 months) were evaluated for activity of peripheral blood natural killer cells. Natural killer-cell cytolytic activity against $\mathrm{K}-562$ tumor-cell targets was examined, as was natural killer-cell suppression of lymphoblastoid B-cell antibody production. In addition, these patients were studied for their ability to generate antitetanus-specific IgG antibody-producing lymphoblastoid B cells following in vivo booster immunization. Crohn's disease patients on 6-mercaptopurine therapy had significant reductions in peripheral blood natural killer-cell activity against K-562 targets compared to normals, disease controls, and Crohn's disease patients not on 6-mercaptopurine. Natural killer-cell suppression of lymphoblastoid B-cell antibody production was likewise decreased in 6-mercaptopurine-treated patients compared to normal controls. In contrast, the in vivo generated lymphoblastoid B-cell antibody responses of Crohn's disease patients on 6-mercaptopurine therapy were not decreased compared to normal, while Crohn's disease patients not on 6-mercaptopurine therapy had significantly impaired IgG antitetanus antibody responses. These findings suggest that 6-mercaptopurine therapy in Crohn's disease affects several lymphoid subpopulations, resulting in a decreased natural killer-cell cytotoxic activity against $\mathrm{K}-562$ target cells and a decreased natural killer-cell ability to suppress lymphoblastoid B-cell antibody production, as well as an improved humoral immune response following tetanus toxoid booster immunization.

KEY WORDS: 6-Mercaptopurine; natural killer cells; suppressor cells; antibody-producing cells; Crohn's disease.

'Department of Medicine, Microbiology and Immunology, UCLA School of Medicine, V.A. Wadsworth Medical Center, Los Angeles, California 90024.

${ }^{2}$ Department of Pathology, University of Michigan Medical Center, Ann Arbor, Michigan 48105.

3Department of Gastroenterology, Lennox Hill Hospital, New York, New York 10021.

To whom correspondence should be addressed at Department of Medicine, Center for the Health Sciences, UCLA School of Medicine, Los Angeles, California 90024.

\section{INTRODUCTION}

A role for immunological mechanisms in the pathogenesis of inflammatory bowel disease has been suspected for years, however, the nature of this involvement remains to be elucidated. The National Cooperative Crohn's Disease Study (1) showed no response to azathioprine therapy of 2 months' duration. In contrast, Present et al. (2) demonstrated that as many as $67 \%$ of patients with Crohn's disease received beneficial responses when placed on medical therapy employing 6-mercaptopurine (6MP). They also noted that clinical responses may be delayed, requiring 3-6 months of therapy. Other clinical studies (3-5) have suggested that azathioprine may be useful as a maintenance treatment in Crohn's disease. The mechanisms by which 6-MP effected a beneficial response in these studies were unknown.

Previous studies by Campbell et al. $(6,7)$ showed that Crohn's disease and ulcerative colitis patients treated with azathioprine, which is metabolized in vivo to 6-MP, had a decrease in killer-cell (K-cell) activity. Shih et al. (8) have demonstrated a profound decrease in the activity of both $K$ cells and natural killer cells (NK cells) in the peripheral blood of multiple sclerosis patients treated with azathioprine. More recently, Prince et al. (9) showed that renal transplant patients on long-term azathioprine therapy had similar decreases in K-cell and NK-cell activity.

In this study, we have examined NK-cell cytotoxic activity in Crohn's disease patients on and off long-term 6-MP therapy to see if decreased activity occurs as in other patient groups. We have also evaluated another aspect of NK-cell activity, the ability to suppress lymphoblastoid B-cell (LB Bcell) antibody production (10), to see if it is likewise decreased. In addition, IgG antitetanus antibody (IgG-Tet)-producing LB B cells were tested in 6- 
were kept at $4^{\circ} \mathrm{C}$ for approximately $12 \mathrm{hr}$ before separating and testing. Samples were warmed to $37^{\circ} \mathrm{C}$ prior to Ficoll-Hypaque centrifugation. To control for effects of cold incubation on NK activity and LB B-cell antibody production, four normal specimens were separated while fresh and following overnight incubation at $4^{\circ} \mathrm{C}$. NK cytotoxic activity at effector to target ratios of $25 / 1$ in fresh $(67 \pm 27 \%)$ and incubated specimens $(60 \pm 30 \%)$ was not significantly different $(P>0.2)$. IgG-Tet production by fresh $(76 \times / \div 3.6 \mathrm{ng} /$ culture $)$ and cold incubated cells $(62 \times / \div 4.3 \mathrm{ng} /$ culture) showed similar results $(P>0.2)$.

\section{Lymphocyte Cultures}

Triplicate cultures containing peripheral blood lymphocytes ( 1.5 million cells) were initiated in a final volume of $1.5 \mathrm{ml}$ in round-bottom culture tubes. The cultures were incubated at $37^{\circ} \mathrm{C}$ with $5 \%$ $\mathrm{CO}_{2}$ for 3 days in a medium consisting of RPMI 1640 buffered with $\mathrm{NaHCO}_{3}$ and supplemented with L-glutamine $(10 \mathrm{~m} M)$, gentamicin $(0.05 \mathrm{mg} / \mathrm{ml})$, and $15 \%$ heat-inactivated fetal calf serum.

\section{Lymphoblastoid-Cell Suppression Cultures}

LB B-cell suppression cultures were performed similarly to lymphocyte cultures except that alpha interferon $(100 \mathrm{U} / \mathrm{ml})$ was added to stimulate NK cell-mediated suppression (10).

\section{ELISA Assays for Antibody Production}

The solid-phase quantitative ELISA tests for IgG-Tet were performed in microtiter plates as previously described (12), substituting alkaline phosphatase-coupled antibody for radioiodinated antibody in the developing antibody step.

\section{NK K-562 Cytotoxicity Assays}

Cytotoxicity tests were performed by ${ }^{51} \mathrm{Cr}$ release in standard 96-well V-shaped microplates (Cooke, Alexandria, VA), in a total volume of 200 $\mu \mathrm{l}$. K-562 tumor-cell targets were labeled with 200 $\mu \mathrm{Ci}$ of $\mathrm{Na}^{51} \mathrm{Cr}$ (New England Nuclear, Boston, MA) for $1 \mathrm{hr}$ at $37^{\circ} \mathrm{C}$ and washed three times. Ten thousand target cells were used in each microtiter well. Effector-to-target ratios of $25 / 1$ and 10/1 were used. After a 4-or 18-hr incubation period, $100 \mu \mathrm{l}$ of supernatant was collected and cytotoxicity was estimated from the released radioactivity according to the formula: [(test release-spontaneous release $) /(80 \%$ of total label-spontaneous release) $] \times 100$.

\section{Statistical Analysis}

Results of IgG-Tet production are expressed as the geometric mean $x / \div$ standard deviation (SD). All other results are expressed as the arithmetic mean $\pm \mathrm{SD}$. The statistical significance of differences between groups was established using the Mann-Whitney $U$ test. The variability of the response was evaluated by chi-square analysis of values lying outside the normal mean \pm 2 SD.

\section{RESULTS}

\section{NK-Cell Activity Against K-562 Targets}

Lymphocytes from both New York Crohn's disease patient groups and normal controls were tested simultaneously for their ability to mediate cytolysis of the standard NK target cell K-562 (Fig. 1). Seven of 12 patients from the New York group had sufficient numbers of lymphocytes after separation to test for NK-cell cytotoxicity. NK-cell activity at

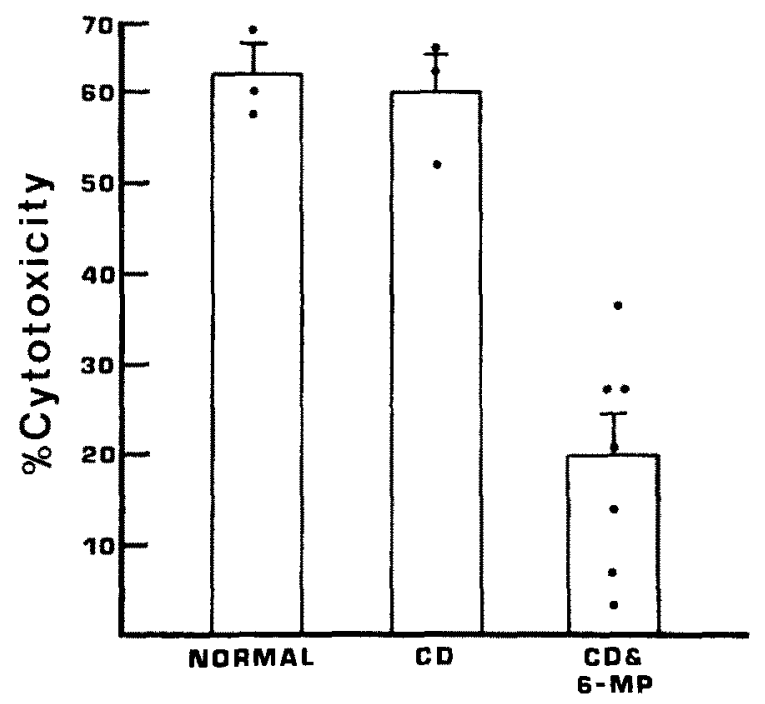

Fig. 1. NK K-562 cytotoxicity of normal controls, Crohn's disease patients not on 6-MP therapy (CD), and Crohn's patients on 6-MP therapy (CD \& 6-MP). All blood specimens were obtained from Dr. Korelitz's practice in New York and all assays were performed simultaneously. Effector-to-target ratios, 25/1. Bars represent arithmetic means $+1 \mathrm{SE}$. 
effector-to-target ratios of $25 / 1$ was not significantly different $(P>0.2)$ in normals $(N=3 ; 62 \pm 6.2 \%$ cytotoxicity) and Crohn's patients not on 6-MP $(N$ $=3 ; 60.3 \pm 7.4 \%$ cytotoxicity). Significant reductions $(P<0.01)$ in the levels of detectable NK-cell activity were observed in patients receiving 6-MP therapy $(N=7 ; 19.9 \pm 12.6 \%$ cytotoxicity). These observations were extended during subsequent testing over a 15-month period at UCLA, using effector-to-target ratios of $25 / 1$ in a standard ${ }^{51} \mathrm{Cr}$ release assay (Fig. 2). Normals $(N=32 ; 33 \pm 13.7 \%$ cytotoxicity) and gastrointestinal disease controls $(N=5 ; 33 \pm 21 \%$ cytotoxicity) were not significantly different $(P>0.2)$. Crohn's disease patients not on 6-MP ( $N=22 ; 25.5 \pm 15 \%$ cytotoxicity) had slightly decreased NK activity compared to normals $(0.04<P<0.05)$ but not compared to disease controls $(P>0.2)$. More impressive were the decreases $(P<0.01)$ in NK-cell activity $(N=10$; $10.7 \pm 10 \%$ cytotoxicity) in patients on 6-MP therapy compared to normals and Crohn's disease patients not on 6-MP. Effector-to-target ratios of 10/1 showed similar results (data not shown). There was little correlation between NK-cell activity and corticosteroid dosage (Fig. 3), with $r=0.15(P>0.2)$ for Crohn's patients both on and off 6-MP therapy.

\section{NK-Cell Suppression of Antibody Production}

To determine whether the subsets of NK cells which are capable of suppressing LB B-cell antibody production in vitro (10) were also affected by 6-MP therapy, simultaneous LB B cell-containing cultures were performed with the addition of interferon, a modulator which stimulates NK-cell suppression of antibody production. The results of these experiments are shown in Fig. 4. Normal lymphocytes $(N=7)$ cultured with interferon produced significantly less IgG-Tet antibody $(34.8 \times 1 \div$ $1.34 \mathrm{ng} /$ culture) than control cultures without interferon $(65.4 \times / \div 1.34 \mathrm{ng} /$ culture; $P<0.01)$. In similar experiments performed on lymphocytes isolated from patients on long-term 6-MP therapy $(N=$ 10), there was an absence of suppression of IgG-Tet production $(P>0.2)$ in cultures with interferon $(69.4 \times / \div 3.2 \mathrm{ng} /$ culture $)$ in comparison to simultaneous cultures without interferon $(87.4 \times / \div 3.3$ ng/culture).

\section{LB B-Cell Generation}

Normal individuals, gastrointestinal disease controls, systemic lupus erythematosus inflammatory

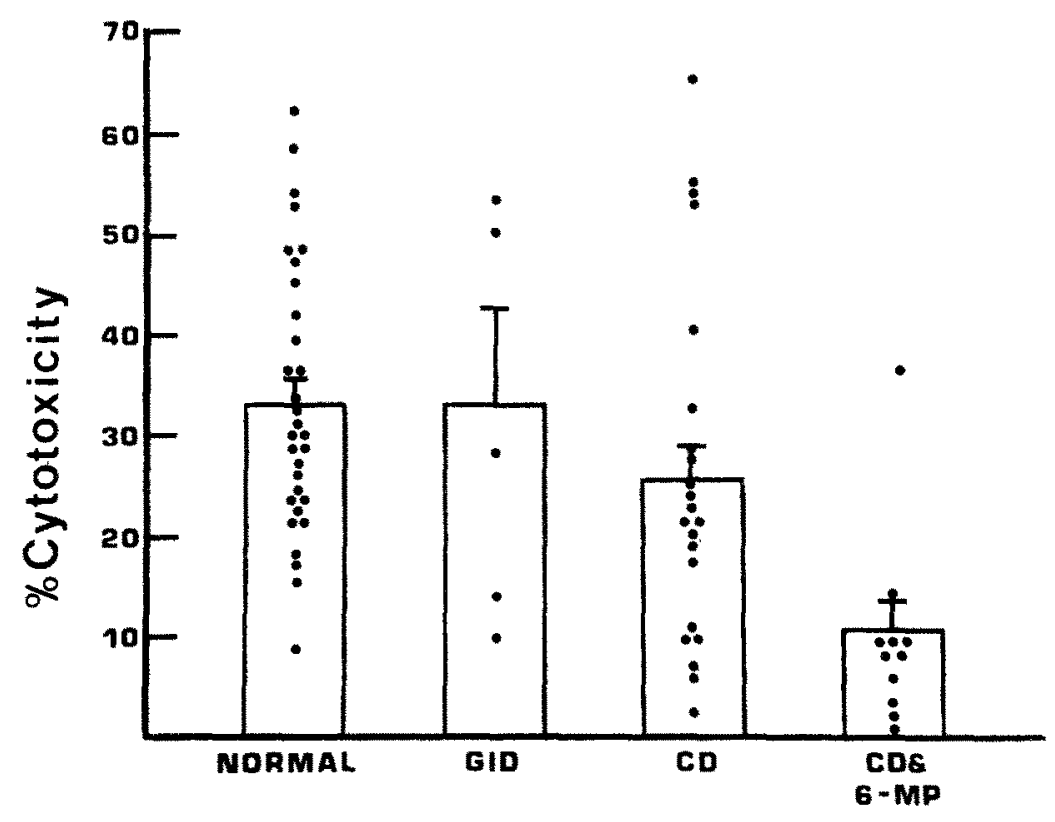

Fig. 2. NK K-562 cytotoxic activity of normal controls, gastrointestinal disease controls (GID), Crohn's disease patients not on 6-MP therapy (CD), and Crohn's patients on 6-MP therapy (CD \& 6-MP). Assays were performed on Los Angeles patients over a 15-month period at UCLA. Effector-to-target ratios, 25/1. Bars represent arithmetic means $+1 \mathrm{SE}$. 


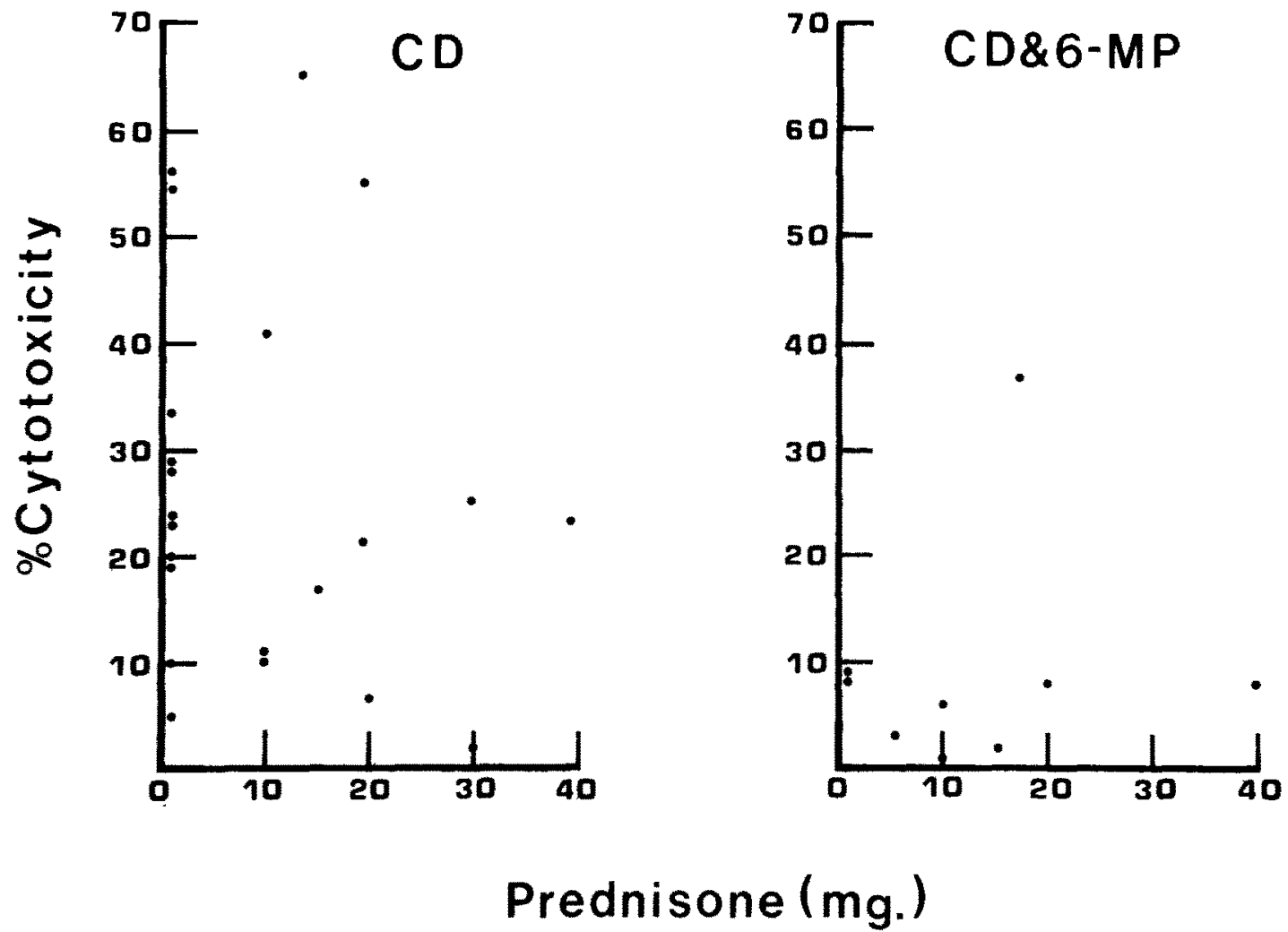

Fig. 3. NK-cell cytotoxic activity against K-562 targets versus prednisone dosage. Dots represent data points of individual patients.

controls, and Crohn's disease patients were booster immunized with tetanus toxoid. All normal individuals $(N=59)$ responded to the immunization with the production of antitetanus LB B-cell activity 7 days after immunization (Fig. 5). There was no apparent difference in IgG-Tet production in the three normal specimens maintained at $4^{\circ} \mathrm{C}$ while being flown from New York to Los Angeles (data not shown). Normal values ranged from 44 to 325 $\mathrm{ng} /$ culture, with a geometric mean of $110 \times / \div 1.6$ ng/culture. Gastrointestinal disease controls $(N=$ 7) responded to immunization, producing $92.8 \times / \div$ $3.2 \mathrm{ng} /$ culture, a value not significantly different from normal $(P>0.2)$. Systemic lupus erythematosus inflammatory controls $(N=9)$ also responded to immunization, producing $86 \times / \div 2.6 \mathrm{ng} /$ culture, again not significantly different from normal $(P>$ $0.1)$. In contrast, Crohn's patients not on 6-MP ( $N$ $=25$ ) had a markedly decreased IgG-Tet LB B-cell response $(32.2 \times / \div 12.1 \mathrm{ng} /$ culture $)$, significantly less than normal $(P<0.002)$. Additional evaluation of the data revealed a wide variability of response in Crohn's patients not on 6-MP, with a range extending from 0 to $3200 \mathrm{ng} /$ culture. Eighty-six percent of these patients were outside the normal mean \pm 2 $\mathrm{SD}\left(\chi^{2}=53, P<0.01\right)$. To assess the effects of 6 MP on the ability of Crohn's disease patients to generate IgG-Tet LB B-cell responses, 16 patients on 6-MP therapy for more than 4 months were evaluated. The LB B-cell responses of these patients were increased $(81.4 \times / \div 2.8 \mathrm{ng} /$ culture $)$ compared to those of Crohn's patients not on 6-MP and were not significantly different from normal $(P$ $>0.2$ ). There was also a decrease in the variability of response, with only $36 \%$ of the patients lying outside the normal mean $\pm 2 \mathrm{SD}$. The variability of response was still significantly increased compared to normal $\left(\chi^{2}=13, P<0.01\right)$ and resembled that of the gastrointestinal disease controls. There was no significant difference in the LB B-cell response of those patients receiving steroids and those not on steroid therapy (13).

\section{DISCUSSION}

Previous investigations of the immunosuppressive effects of azathioprine (6-9) have shown that its major effects following long-term in vivo therapy 


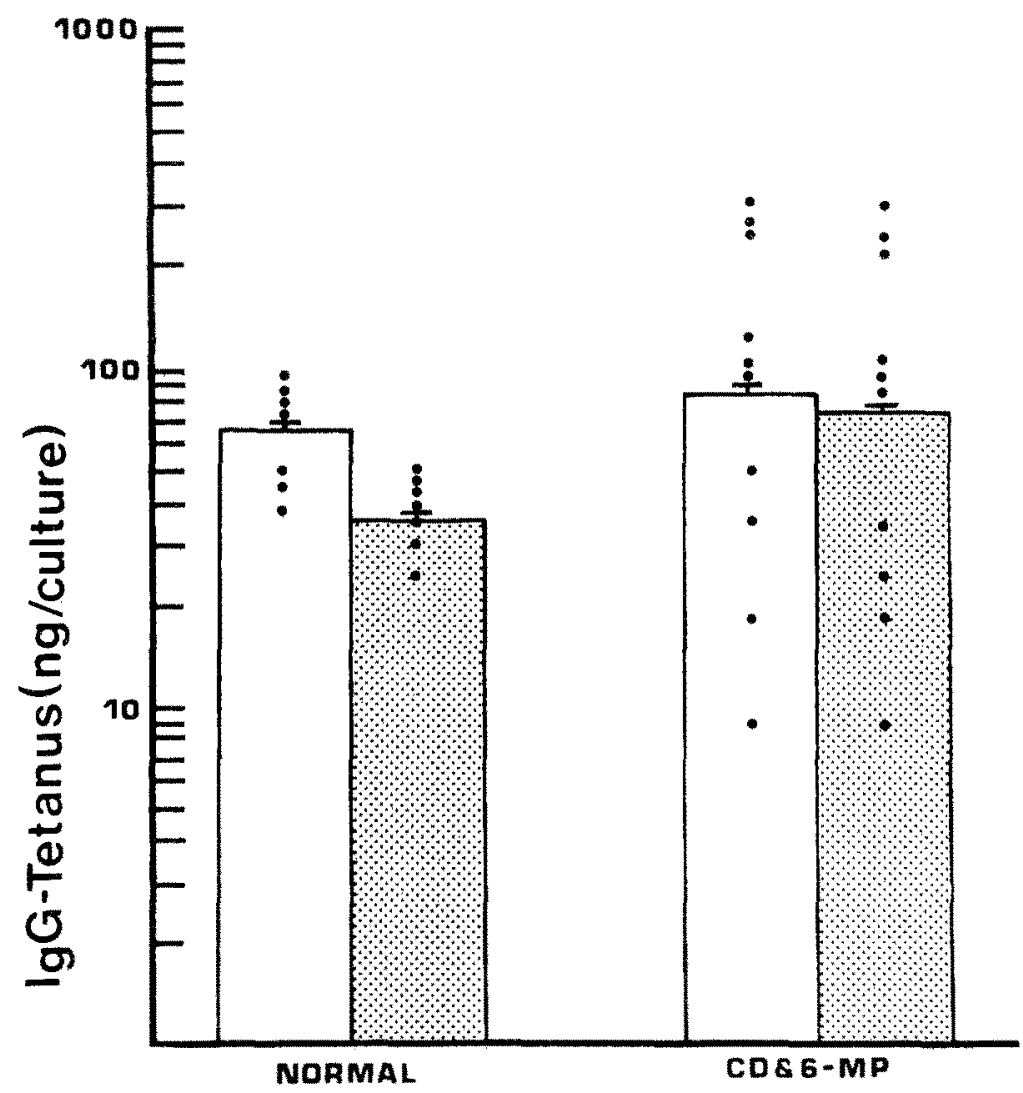

Fig. 4. NK-cell suppression of LB B-cell IgG-Tet antibody production in normals and Crohn's disease patients on 6-MP therapy (CD \& 6-MP). Shaded bars represent simultaneous cultures with interferon added to stimulate NK cellmediated suppression. Bars represent geometric means +1 geometric SD.

are on NK- and K-cell cytotoxic lymphocytes. These cells are large granular lymphocytes which are quite similar phenotypically and, in fact, may be identical or overlapping populations which are cytotoxic by different mechanisms (14). Initially Campbell et al. $(6,7)$ demonstrated that inflammatory bowel disease patients treated with azathioprine had decreased $\mathrm{K}$-cell activity as measured in an antibody-dependent cytotoxicity (ADCC) assay. Shih et al. (8) extended the in vivo evaluation of the effect of azathioprine on immune functions by prospectively studying a group of multiple sclerosis patients who had been treated with similar doses of this drug. The results from this study demonstrated a selective and profound decrease in the peripheral blood activity of $\mathrm{K}$ and NK cells. Other parameters tested, i.e., the T- and B-cell numbers and the phytohemagglutinin (PHA) and concanavalin A (Con A) proliferative responses, were unaffected. This reduced $\mathrm{K}$ - and NK-cell activity was due to a depletion of circulating cells and was not due to a decrease in cell function. A temporal delay in the loss of K-and NK-cell activity was observed, with an initial decline seen at 1 month after the initiation of therapy, followed by a nadir at 4-5 months. Interestingly, this time course was similar to that of clinical improvement seen in Crohn's disease patients by Present $e t$ al. (2). More recently, Prince $e t$ al. (9) compared the immunosuppressive effects of long-term azathioprine versus prednisone therapy in renal transplant patients. They demonstrated that azathioprine, and not prednisone, suppressed NK and ADCC activity.

The NK activity of peripheral blood lymphocytes in our Crohn's disease patients was slightly decreased in comparison to that of normals but not that of gastrointestinal disease controls. Previous investigators have found both normal (15) and decreased $(16,17)$ levels of peripheral blood NK-cell cytotoxic activity in inflammatory bowel disease. Differences in methodology, as well as differing levels of disease activity, may explain this variation 


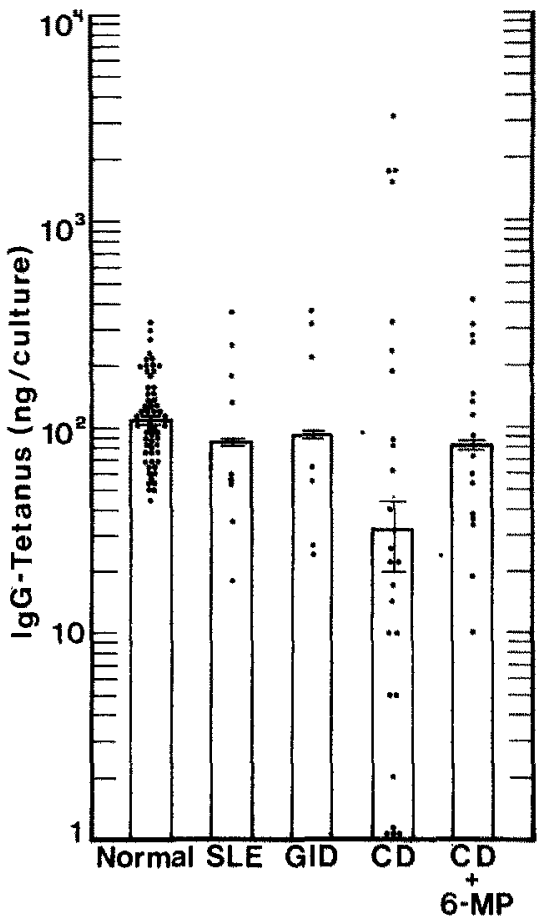

Fig. 5. LB B-cell IgG-Tet antibody production 7 days after immunization by normals, systemic lupus erythematosus inflammatory controls (SLE), gastrointestinal disease controls (GID), Crohn's disease patients not on 6-MP (CD), and Crohn's disease patients on 6-MP therapy (CD + 6-MP). Bars represent geometric means + 1 geometric $\mathrm{SD}$.

in NK-cell cytotoxic activity. Ginsberg et al. (16) tested NK cytotoxicity following overnight culture of lymphocytes, which results in the activation of normal NK-cell cytotoxicity. Our patients as well as those of James et al. (15) had minimal disease activity in comparison to those in studies which demonstrated decreased NK cytotoxicity activity $(16,17)$.

We have demonstrated that decreased NK-cell cytotoxic activity as well as decreased NK-cell suppression of LB B-cell antibody production occurs in the peripheral blood of Crohn's disease patients on long-term 6-MP therapy. These antibody-suppressing cells in normals are stimulated by interferon, are subject to cold target competition by K-562 cells, and express the Leu 11 phenotype (10). They may represent a subset of NK cells.

Although the NK activity against K-562 targets is normal or slightly decreased in Crohn's disease patients not on 6-MP, it is possible that a subset of NK cells directed against LB B cells is increased in Crohn's patients, contributing to their abnormal antibody responses to tetanus. A second possibility is that greater activity of NK cells is induced by immunization in Crohn's patients than in normals. These hypotheses are currently being pursued.

There was little correlation between corticosteroid dosage and NK cytotoxic activity. This confirms the lack of corticosteroid effect on NK-cell cytotoxicity seen by Prince et al. (9).

The relationship of these findings to the clinical efficacy of 6-MP therapy is uncertain. Recent studies, however, have demonstrated a role for $\mathrm{K}$ and NK cells in intestinal tissue. Animal experiments using functional and monoclonal analysis have shown the presence of large granular lymphocytes (NK and $\mathrm{K}$ cells) among the small intestine and colonic intraepithelial and Peyèr's patch cells (1820). Reynolds et al. have demonstrated that large granular lymphocytes injected into rats preferentially home to the intestines (18). Additional studies in our laboratory have demonstrated the presence of endogenous as well as interferon-activatable NK cells in normal intestine (21) and, more recently, have suggested that increased NK-cell cytotoxic activity may be seen in Crohn's disease intestine (22). Britton et al. (23) have previously shown an increase in $\mathrm{K}$ cells in the mesenteric lymph nodes of Crohn's disease patients. Shorter et al. (24) have isolated mononuclear cells from colonic mucosa of inflammatory bowel disease patients which are cytotoxic for autologous colonic epithelial cells, possibly by a K-or NK-cell mechanism. Further studies of NK cells at the intestinal level in Crohn's patients are required to determine the existence of NK-cell cytotoxic or antibody suppression activity in this compartment and its relationship to the clinical efficacy of 6-MP therapy.

In a previous study, we demonstrated that many Crohn's disease patients have defects in their humoral immune system in that they fail to generate normal levels of IgG-Tet antibody-producing LB Bcell activity following in vivo immunization (13). In addition, these patients failed to show increases in serum levels of tetanus toxoid-specific IgG antibody. The present study adds new findings to the abnormal LB B-cell responses to tetanus recently associated with Crohn's disease patients not on 6MP therapy. 6-MP-treated Crohn's patients made a significant IgG-Tet LB B-cell response following immunization. In addition, the variability of the response was decreased, with fewer patients lying outside the normal range. This improved LB B-cell response to tetanus may be related to the decreased NK-cell suppressor activity seen in these patients. 
These suppressor cells may represent a previously unrecognized lymphoid subpopulation which is sensitive to 6-MP therapy. Further prospective evaluations of these cells in inflammatory bowel disease patients will help to determine the sensitivity of these NK suppressor cells to 6-MP therapy as well as the relationship between improved humoral responsiveness and clinical efficacy.

\section{ACKNOWLEDGMENTS}

This work was partially supported by USPHS Grants AI-16694, CA-34899, and AM-27806, a grant from the Blinder Crohn's Disease Research Foundation, a grant from the National Foundation for Ileitis and Colitis, and a grant from Reach Out from Youth with Inflammatory Bowel Disease. S.T. is the recipient of a V.A. Clinical Investigators Award. The authors wish to thank Birgitte Keld and Adrene Niederlehner for excellent technical assistance and the staff at the Medical Immunology Research Laboratory at UCLA, who performed some of the NK-cell assays.

\section{REFERENCES}

1. Summers R, Switz D, Sessions J, Becktel J, Best W, Kern F, Singleton J: National Cooperative Crohn's Disease Study: Results of drug treatment. Gastroenterology 77:847-869, 1979

2. Present D, Korelitz B, Wisch N, Glass J, Sachar D, Pasternack B: Treatment of Crohn's disease with 6-mercaptopurine: A long term randomized double blind study. $\mathrm{N}$ Engl J Med 302:981-987, 1980

3. O'Donoghue D, Dawson A, Powell-Tuck J, Brown R, Lennard-Jones J: Double blind withdrawal trial of azathioprine as maintenance treatment for Crohn's disease. Lancet $2: 955-957,1978$

4. Willoughby J, Kumar P, Beckett J, Dawson A: Controlled trial of azathioprine in Crohn's disease. Lancet 2:944-946, 1971

5. Rosenberg J, Levin B, Wall A, Kirsener J: A controlled trial of azathioprine in Crohn"s disease. Digest Dis 20:721-726, 1975

6. Campbell A, Skinner I, Hersey P, Roberts-Thompson P, MacLennan I, Truelove S: Immunosuppression in the treatment of inflammatory bowel disease. I. Changes in lymphoid subpopulations in the blood and rectal mucosa following cessation of treatment with azathioprine. Clin Exp Immunol $16: 521-533,1974$

7. Campbell A, Skinner J, MacLennan I, Hersey P, Waller A, Wood $\mathfrak{J}$, Jewell D, Truelove S: Immunosuppression in the treatment of inflammatory bowel disease. II. The effects of azathioprine on lymphoid cell populations in a double blind trial in ulcerative colitis. Clin Exp Immunol 24:249-258, 1976

8. Shih W, Ellison G, Myers L, Durkos-Smith D, Fahey J: Locus of selective depression of human natural killer cells by azathioprine. Clin Immunol Pathol 23:672-681, 1982

9. Prince H, Ettinger R, Dorey F, Fine R, Fahey J: Azathioprine suppression of natural killer activity and antibodydependent cellular cytotoxicity in renal transplant patients. $J$ Clin Immunol 4:312-318, 1984

10. Brieva J, Targan S, Stevens R: NK and T cell subsets regulate antibody production by human in vivo induced lymphoblastoid B cells. J Immunol 132:611-615, 1984

11. Boyum A: Isolation of mononuclear cells and granulocytes from human blood. Scand J Clin Lab Invest 21 (Suppl 97): $77-89,1968$

12. Stevens R, Saxon A: Immunoregulation in humans: Control of antitetanus antibody production after booster immunization. J Clin Invest 62:1154-1160, 1978

13. Stevens $\mathbf{R}$, Oliver $\mathbf{M}$, Brogan $\mathrm{M}$, Hiserodt $\mathrm{J}$, Targan $\mathrm{S}$ : Defective generation of tetanus specific antibody producing $B$ cells following in vivo immunization of Crohn's disease and ulcerative colitis patients. Gastroenterology (in press)

14. Parillo J, Fauci A: Comparison of the effector cells in human spontaneous cellular cytotoxicity and antibody dependent cellular cytotoxicity: Differential sensitivity of effector cells to in vivo corticosteroids. Scand J Immunol 8:99-107, 1978

15. James S, Neckers L, Graeff A, Cossman J, Balch C, Strober W: Suppression of immunoglobulin synthesis by lymphocyte subpopulations in patients with Crohn's disease. Gastroenterology 86:1510-1518, 1984

16. Ginsburg C, Dambrauskas J, Ault K, Falchuk Z: Impaired natural killer cell activity in patients with Crohn's disease: Evidence for a qualitative defect. Gastroenterology 85:846851,1983

17. Auer I, Ziemer E, Sommer H: Immune status in Crohn's disease. V. Decreased in vitro natural killer cell activity in peripheral blood. Clin Exp Immunol 42:41-49, 1980

18. Ward J, Argilan F, Reynolds C: Immunoperoxidase localization of large granular lymphocytes in normal tissues and lesions of athymic nude rats. J Immunol 131:132-139, 1983

19. Tagliabue A, Befus A, Clark D, Bienenstock J: Characteristics of natural killer cells in the murine intestinal epithelium and lamina propria. J Exp Med 155:1785-1796, 1982

20. Tagliabue A, Luini W, Soldateschi D, Boraschi D: Natural Killer activity of gut mucosal lymphoid cells in mice. Eur J Immunol 11:919, 1981

21. Targan S, Britvan L, Kendall R, Vimadalal S, Soll A: Isolation of spontaneous and interferon inducible natural killer like cells from human colonic mucosa: Lysis of lymphoid and autologous epithelial target cells. Clin Exp Immunol 54:14-22, 1983

22. Oliver M, Stevens R, Targan S: Natural killer cell activity, suppression of natural killer killing and spontaneous antibody secretion are evident in normal and inflammatory bowel disease colonic mucosa if lymphoid cells are promptly isolated. Gastroenterology 85:1200, 1984

23. Britton S, Eklunda E, Bird A: Appearance of killer (K) cells in the mesenteric lymph nodes in Crohn's disease. Gastroenterology 75:218-220, 1978

24. Shorter R, McGill D, Bahn R: Cytotoxicity of mononuclear cells for autologous colonic epithelial cells in colonic diseases. Gastroenterology 96:13-22, 1984 